\title{
Comparison of Two Segmentation Methods for Mammographic Image
}

\author{
Priyanka Jagya \\ ECE, Hindu College of Engg. \\ Sonepat, India
}

\author{
R.B. Dubey \\ ECE, Hindu College of Engg. \\ Sonepat, India
}

\begin{abstract}
Currently mammography is the method of choice for early detection of breast cancer. The image segmentation aims to separate the structure of interest objects from background and other objects. Detection of breast cancer is a very crucial step in mammograms and therefore needs an accurate and standard technique for breast tumor segmentation. In the last few years, a number of algorithms have been published in the literature. Each one has their own merits and de-merits. Fuzzy-level set and wavelet with level set is proposed to facilitate mammogram image segmentation. The existing active contour models can be classified as edge-based models and regionbased model. In fuzzy level set, edge based active contour model is used while, in wavelet with level set, a region-based image segmentation technique using active contours with signed pressure force function is used. Furthermore, after evaluating various parameters wavelet with level set is considered to be better than fuzzy level set, as segmentation of mass area is more effective having less error value and it shows higher PSNR as compared to other method.
\end{abstract}

\section{Keywords}

Segmentation, fuzzy- level set, wavelet with level set, active contour, region of interest.

\section{INTRODUCTION}

Modern medical imaging modalities generate larger and larger images which simply cannot be examined manually. This drives the development of more efficient and robust image analysis methods, tailored to the problems encountered in medical images. Image segmentation is the problem of partitioning an image in a semantically meaningful way. For many applications, segmentation reduces to finding an object in an image. This involves partitioning the image into two classes of regions - either object or background. Segmentation is taking place naturally in the human visual system. Literature is full of experts on detecting patterns, lines, edges and shapes, and making decisions based upon the visual information. It is simply not feasible in practice to manually process all the images. Instead, algorithms has been designed which look for certain patterns and objects of interest and put them to our attention. Segmentation methods should be able to provide fast computation, avoid over segmentation and produce accurate and intuitive segmentations. The fuzzy level set method demonstrates those qualities. Therefore, we choose to extend it to segment mammogram images. Segmentation tends to be the first essential and crucial step of most medical image analysis tasks, since once a region of interest is segmented, one can deduce shape, appearance and structural features about the region. These qualities in turn can be used for diagnosis aid or treatment evaluation. There are many applications of segmentation. For example, we develop a segmentation method for detecting the breast region in mammograms, which is the first step in a Computer-Aided Detection (CAD) process. Detecting the breast region allows the radiologist to automatically zoom in on the breast, hence removing the background and confining the area of which the radiologist should look at and diagnose $[1,2]$.

The many of research works conducted in the area of breast cancer detection and classification much university, commercial institution and research centers are focused on this issue because of the fact that breast cancer is becoming the most common form of cancer disease of today's female population [3]. Breast cancer is one of the major causes of death among women. Cancer disease begins in the cells of the human body, which is generated by abnormal division of those cells. There are two types of cancer, benign tumors are not cancerous and malignant tumors are cancerous [4]. Breast cancer screening is vital to detecting breast cancer [5, 6]. Currently, mammography imaging is most efficient imaging technique. The earlier the cancer is detected, the better the chance that a proper treatment can be prescribed [7].

\section{RELATED WORK}

Nowadays, X-ray mammography is method of choice for early detection of breast cancer [8]. Computer-aided diagnosis (CAD) systems are the double reading substitute for radiologist in diagnosis of breast cancer [9]. Mammogram image segmentation is a very popular research topic in medical image processing. The diversity of every slice of image has given rise to various different approaches to solve the segmentation problem. Similarly, many survey works have been conducted by fellow researchers to determine better methods and develop future optimal techniques based on existing methods. Different methods [10] have reviewed to identify the practical problems associated in implementing various methods to achieve better segmentation results.

A technique [11] employs fuzzy C-mean (FCM), k-mean (KM), marker controlled watershed segmentation (MCWS) and region growing (RG) for the detection and segmentation of masses in mammographic images. FCM is the unsupervised pixel classification technique that aims at dividing image pixels into optimal number of clusters. It was first proposed by Dunn et al [12]. In general, pixels in a cluster have high degree of similarity than those in different clusters. This degree of belongings is represented by a fuzzy membership function. K-mean is known as one of the simplest unsupervised learning algorithms that are used to partition an image into k-clusters [13].The objective of this algorithm is to minimize the objective function that represents the total intracluster variance. Watershed algorithm is considered as the powerful tool for image segmentation. It plays an important role in machine vision, video image segmentation and image analysis [14]. Vincent [15] proposed the novel approach for finding the watershed lines by using the immersion simulation algorithm. Region growing method relies on the propagation 
of an initial seed point according to pre-defined homogeneity criterion [16].

Segmentation using threshold, level set, morphological techniques, fuzzy $\mathrm{C}$-mean clustering and $\mathrm{k}$-mean clustering has been established [17]. A new approach is presented for computer aided detection of micro calcification clusters in digital mammograms [18]. The result of watershed segmentation lies entirely on the image contrast. Watershed algorithm can generate under or over segmentation on badly contrast images. To overcome these deficiencies of watershed algorithm a preprocessing step using curve let or wavelet transform is performed.

Another method [19] is proposed an application of wavelet based K-mean algorithm in mammogram segmentation. Discrete wavelet transform is used to extract the high level details from MRI images. Wavelets provide frequency information as well as time-space localization to make noise free algorithm. Reference [20] proposes a mammogram image segmentation using fuzzy hybrid with particle swarm optimization (PSO). In this, the FCM clustering algorithm is used for segmentation which is further enhanced by using PSO optimization algorithm. This causes increase in resultant accuracy with higher quality.

Cass et al. introduced an active contour method to segment ROI in the computer vision and image processing applications [21]. In this method, a curve is evolved under a force by minimizing the energy until it stops at the object boundary. The energy functional is normally dependent on different characteristics like curvature, image gradient, and image statistical information. The existing active contour models can be classified into two categories: edge-based models [21-24] and region-based models [25-33]. These two types of models have their own advantages and disadvantages. Compared to an edge-based model, a region-based model performs better on images with weak or blurred edges. A region-based model is not sensitive to initialization of the level set function and can recognize object's boundaries efficiently. Therefore, region-based models, especially Chan and Vase (CV) model [23], have been widely applied for image segmentation. Although, a region-based model is better than an edge-based model in some aspects but it still has limitations. The traditional region-based models [23, 25] can't segment intensity inhomogeneous objects in an image.

A technique used for occasionally reinitializing a level set function to a signed distance function during the evolution. From a practical viewpoint, the re-initialization process can be quite convoluted and expensive and can have various side effects [34]. Zhang et al. proposed the active contours with selective local or global segmentation method, which uses a Gaussian kernel to regularize the level function after each iteration step. It not only regularizes the level set but also removes the need of computationally expensive reinitialization. An edge-based active contour model gives very poor results for the images with intense noise and weak edges, while on the other hand a region-based model gives no satisfactory result for the images with the intensity in homogeneity. The traditional region-based model [23] cannot properly segment image with intensity in homogeneity because it cannot differentiate small intensity differences between two consecutive regions and cannot detect weak object boundaries.

Segmentation of regions of interest is a well-known problem in image segmentation. Reference [35] presents a regionbased image segmentation technique using active contours with signed pressure force (SPF) function. This algorithm traces high intensity or dense regions in an image by evolving the contour inwards. In medical image modalities these high intensity or dense regions refer to tumor, masses, or dense tissues. Level set method, which are established on dynamic implicit interfaces and partial differential equations (PDE) have been shown to be effective for medical image segmentation [36-38]. To employ these methods a lot of intense computational requirement and complex regulation of controlling parameters is the hurdle [39]. Research is therefore oriented to facilitating the manipulation, while enhancing the quality of segmentation [42, 37, 39-41]. There have been many hybrid intelligent systems using fuzzy clustering to facilitate level set segmentation [36, 37, 40-41]. In short, such algorithm employ fuzzy clustering based on image intensity for initial segmentation and employ level set methods for object refinement by tracking boundary variation. Fuzzy clustering [36] by approximately delineating tumor boundaries, not only relieves manual intervention, but also accelerates level set optimization. Ho and Sufi proposed to regularize level set evolution locally by fuzzy clustering, in order to alleviate the problems of noise sensitivity and weak boundaries $[37,40,41]$.

Reference [43] utilizes integrating spatial fuzzy clustering with level set methods for automated medical image segmentation. The performance of level set segmentation is subject to appropriate initialization and optimal configuration of controlling parameters, which requires substantial manual intervention. A fuzzy level set algorithm is proposed to facilitate medical image segmentation. It is able to directly evolve from the initial segmentation by spatial fuzzy clustering. The controlling parameters of level set evolution are also estimated from the results of fuzzy clustering. Moreover, the fuzzy level set algorithm is enhanced with locally regularized evolution. Wavelet transform-based methods offer a natural framework for providing multi-scale image representations that can be separately analyzed. In these methods two types of segmentation is used for mammogram to detect tumor. Coarse segmentation is implemented by using wavelet based histogram threshold where the threshold value is chosen by performing in wavelet based analysis of PDF of wavelet transformed images at different channels and second one is fine segmentation which is obtained by choosing threshold by using windowing method. The wavelet based adaptive windowing method is effective to segment the tumor in mammograms and it can also be used in other segmentation applications. This method has limitation of more computational time as compared to other methods [44].

\section{MATERIALS AND METHODOLOGY}

\subsection{Mammogram Image Database}

Using Joyce-Loeb scanning microdensitometer films taken from the UK National Breast Screening Program me have been digitized to 50 micron pixel edge. The database contains 322 digitized films and is available on $2.3 \mathrm{~GB} 8 \mathrm{~mm}$ (Exabyte) tape. Database contains various information i.e. character of background tissue, class of abnormalities present and severity of abnormality present. The database has been reduced to a 200 micron pixel edge and padded/clipped so that all the images are of $1024 \times 1024$. Mammographic images are available via the Pilot European Image Processing Archive (PEIPA) at the University of Essex. 


\subsection{Algorithm used}

\subsubsection{Fuzzy with Level Set}

In fuzzy clustering, a pre-defined cost function is minimized adaptively by estimating the centroid and scope of each subclass thus the fuzzy clustering is used as a kind of adaptive threshold. One of the most popular algorithms in fuzzy clustering is fuzzy c-means and has been widely applied to medical problems [47].

The k-means algorithm seeks to assign $\mathrm{N}$ objects, based on their attributes, into $\mathrm{K}$ clusters $(\mathrm{K} \leq \mathrm{N})$. For medical image segmentation, $\mathrm{N}$ equals the number of image pixels $N_{x} * N_{y}$.Standard k-mean clustering attempts to minimize the cost function.

$j=\sum_{m=1}^{K} \sum_{n=1}^{N}\left[i_{n}-v_{m}\right]^{2}$

where $i_{n}$ is the specific image pixel, $v_{m}$ is centroid of the $\mathrm{m}^{\text {th }}$ cluster.

Thus the inter-cluster variations are maximizing using k-mean algorithm, but the intra-cluster variations are minimized. In kmean clustering, every object is limited to one and only one of $\mathrm{k}$ clusters. In contrast, membership function $\mu_{m n}$ is utilized in case of FCM to indicate the degree of membership of the nth object to the $m_{t h}$ cluster. The cost function in an FCM is

$j=\sum_{n=1}^{N} \sum_{m=1}^{C} \mu_{m n}^{l}\left[i_{n}-v_{m}\right]^{2}$

Where, $l(>1)$ is a parameter controlling the fuzziness of the resultant segmentation. The membership functions $\mu_{m n}$ and the centroids $v_{m}$ are updated iteratively,

$$
\begin{gathered}
\mu_{m n}=\frac{\left[i_{n}-v_{m}\right]^{\frac{-2}{l-1}}}{\sum_{k=1}^{C}\left[i_{n}-v_{k}\right]^{\frac{-2}{l-1}}} \\
v_{i}=\frac{\sum_{n=1}^{N} \mu_{m n}^{l} i_{n}}{\sum_{n=1}^{N} \mu_{m n}^{l}}
\end{gathered}
$$

The standard FCM algorithm is optimized when high membership values are assigned to the pixels close to the centroid while those that are far away are assigned low values.

In contrast to FCM using pixel classification, level set method utilizes dynamic variation boundaries for image segmentation. Segmentation of an image by means of active contours is a well known approach but instead of parametric characterization of active contours, level set methods embed them into a time dependent partial differential equation (PDE) function $\varnothing(\mathrm{t}, \mathrm{x}, \mathrm{y})$.Note, that the inclusion of the time variable $t$, leads to a higher dimensional level set function $\varnothing$, which incurs an additional computation, but has many practical advantages [43].

In particular, the evolution of $\varnothing$ is totally determined by the numerical level set equation

$$
\begin{aligned}
& \frac{\partial \phi}{\partial t}+\mathrm{F}|\nabla \phi|=0 \\
& \phi(0, \mathrm{x}, \mathrm{y})=\phi_{0}(\mathrm{x}, \mathrm{y})
\end{aligned}
$$

where $|\nabla \varnothing|$ denotes the normal direction, $\phi_{0}(\mathrm{x}, \mathrm{y})$ is the initial contour and $\mathrm{F}$ represents the comprehensive forces including the internal force from the interface geometry(e.g., mean curvature, contour length and area) and the external force from image gradient.

The advancing force $\mathrm{F}$ has to be regularized by an edge indication function, $\mathrm{g}$ in order to stop level stop evolution near the optimal solution.

$$
g=\frac{1}{1+\left|\nabla\left(\mathrm{G}_{\sigma} * \mathrm{I}\right)\right|^{2}}
$$

Where $\left(G_{\sigma} * \mathrm{I}\right)$ stands for the convolution of the image I with a smoothing Gaussian $\operatorname{kernel} G_{\sigma}$, and $\nabla$ denotes the operation for an image gradient. The function $\mathrm{g}$ is near zero in variation boundaries, but positive otherwise.

A popular formulation for level set segmentation is:

$$
\frac{\partial \phi}{\partial t}=\mathrm{g}|\nabla \phi|\left(\operatorname{div}\left(\frac{\nabla \phi}{|\nabla \phi|}\right)+\mathrm{v}\right)
$$

where, $\operatorname{div}\left(\frac{\nabla \phi}{|\nabla \phi|}\right)$ approximates the mean curvature k, and $\mathrm{v}$ is a balloon force.

One of the biggest challenges in level set segmentation is the intensive computation. In order to overcome these challenges, a fast level set formulation is proposed [43]

$$
\frac{\partial \phi}{\partial t}=\mu \xi(\phi)+\xi(\mathrm{g}, \phi)
$$

Where the first term $\xi(\phi)$ at the right side is a penalty momentum of $\varnothing$, deviating from the signed distance function

$$
\xi(\phi)=\nabla \phi-\operatorname{div}\left(\frac{\nabla \phi}{|\nabla \phi|}\right)
$$

The second term $\xi(\mathrm{g}, \phi)$ incorporates image gradient information by

$$
\xi(\mathrm{g}, \phi)=\lambda \partial(\phi) \operatorname{div}\left(\mathrm{g} \frac{\nabla \phi}{|\nabla \phi|}\right)+\operatorname{vg} \partial(\phi)
$$

where, $\partial(\phi)$ denotes the Dirac function. The constants $\mu, \lambda$ and $\mathrm{v}$ control the individual contributions of these terms. 
In essence, the term $\xi(\mathrm{g}, \phi)$ attracts $\varnothing$ towards the variation in boundary, which is similar to the standard level set methods. The penalty term $\xi(\phi)$ forces to approach the genuine signed distance function automatically, which has important advantages. First, the new algorithm eliminates the computationally expensive re-initialization for signed distance functions [43].

Both FCM algorithms and level set methods are generalpurpose computational models that can be applied to problem of any dimension. Thus in case of fuzzy level set ,the results of fuzzy clustering are utilized to initiate the level set segmentation, controlling parameters are estimated and level set evolution is regularize. Suppose the component of interest in an FCM results is $\mathrm{R}_{\mathrm{k}}:\left\{\mathrm{r}_{\mathrm{k}}=\mu_{\mathrm{nk}}, n=x * \mathrm{~N}_{y}+y\right\}$.It is then convenient to initiate the level set function as

$$
\phi_{0}(\mathrm{x}, \mathrm{y})=-4 \varepsilon\left(0.5-\mathrm{B}_{k}\right)
$$

where ' $\varepsilon$ ' is a constant regulating the Dirac function, The Dirac function is then defined as

$$
\begin{array}{ll}
\delta_{\varepsilon}(\mathrm{x})=0 & |\mathrm{x}|>\varepsilon \\
\frac{1}{2 \varepsilon}\left[1+\cos \left(\frac{\pi \mathrm{x}}{\varepsilon}\right)\right] & |\mathrm{x}| \leq \varepsilon
\end{array}
$$

$B_{k}$ is a binary image obtained from,

$$
\mathrm{B}_{k}=\mathrm{R}_{k} \geq \mathrm{b}_{0}
$$

where, $b_{0} \in(0,1)$ is an adjustable threshold.

The fuzzy level set algorithm takes the degree of membership of each image pixel $\mu_{k}$ as the distance to the specific component of interest $R_{k}$. The balloon force is enhanced to pull or push the dynamic interface adaptively towards the object of interest.

$$
G\left(\mathrm{R}_{k}\right)=1-2 \mathrm{R}_{k}
$$

The resultant balloon force is a matrix with a variable pulling or pushing force at each image pixel. In other words, the level set function will be attracted towards the object of interest regardless its initial position. Thus the equation is transformed as:

$$
\xi(\mathrm{g}, \phi)=\lambda \delta(\phi) \operatorname{div}\left(\mathrm{g} \frac{\nabla \phi}{|\nabla \phi|}\right)+\mathrm{gG}\left(\mathrm{R}_{k}\right) \delta(\phi)
$$

In this fuzzy level set, edge based active contour model is used. The edge-based model builds an edge stopping function using image edge information, which enforces the evolution of contour towards the object boundary. A balloon force term is used in the contour evolution process, which helps the contour to shrink or expand. The selection of an accurate balloon force is main problem in this method .Furthermore, for the images with intense noise or weak edges, the edge stopping function based on the image gradient can hardly stop at correct boundaries [35]. The methodology for mammogram image segmentation using fuzzy level set is shown in Fig. 1.

\subsubsection{Wavelet with Level Set}

The wavelet transform is important to provide a compact description of images that are limited in time and it is very useful in description of edges and line that are highly localized [19]. A 1-level wavelet transform of a discrete image ' $\mathrm{f}$ ' can be done by using the following two steps:

Step1: perform a 1-level, 1D wavelet transform on each row of $f$, thereby producing a new image.

Step2: On the new image obtained from step1, perform the same 1D wavelet transform on each of its columns.

It can be easily shown that steps 1 and 2 could be done in reverse order and result would be the same. A 1-level wavelet decomposition of an image $f$ can be defined as follows:

$\mathrm{f} \rightarrow\left(\frac{\mathrm{h}^{1}}{a^{1}} \frac{d^{1}}{v^{1}}\right)$

where $h^{1}, a^{1}, d^{1}$ and $v^{1}$ are sub images each have $\frac{M}{2}$ rows and $\frac{N}{2}$ columns, where $\mathrm{M}$ and $\mathrm{N}$ are the number of rows and columns respectively. The sub image $a^{1}$ is created by computing trends along rows of ' $\mathrm{f}$ ' followed by computing trends along columns; so it is an averaged, lower resolution version of the image $f$. This sub image is referred as measure of horizontal fluctuation.

The sub image $v^{1}$ is similar to $h^{1}$, except that the roles of horizontal and vertical are reversed. This sub image is referred as measure of vertical fluctuation. Finally, there is the first diagonal fluctuation $d^{1}$.This sub image tends to emphasize diagonal features, because it is created from fluctuations along both rows and columns.

In wavelet with level set method first, apply wavelet transform on the mammogram image to obtain wavelet decomposed image resulting in four sub bands. These are the LL (Lower resolution version of image), LH (Horizontal edge data), HL (Vertical edge data), \& HH (Diagonal edge data) sub bands representing approximation, horizontal, vertical and diagonal components in the form of coefficients, respectively. LL sub band contains low level and the other three (LH, HL, and $\mathrm{HH}$ ) contain high level details [19]. 


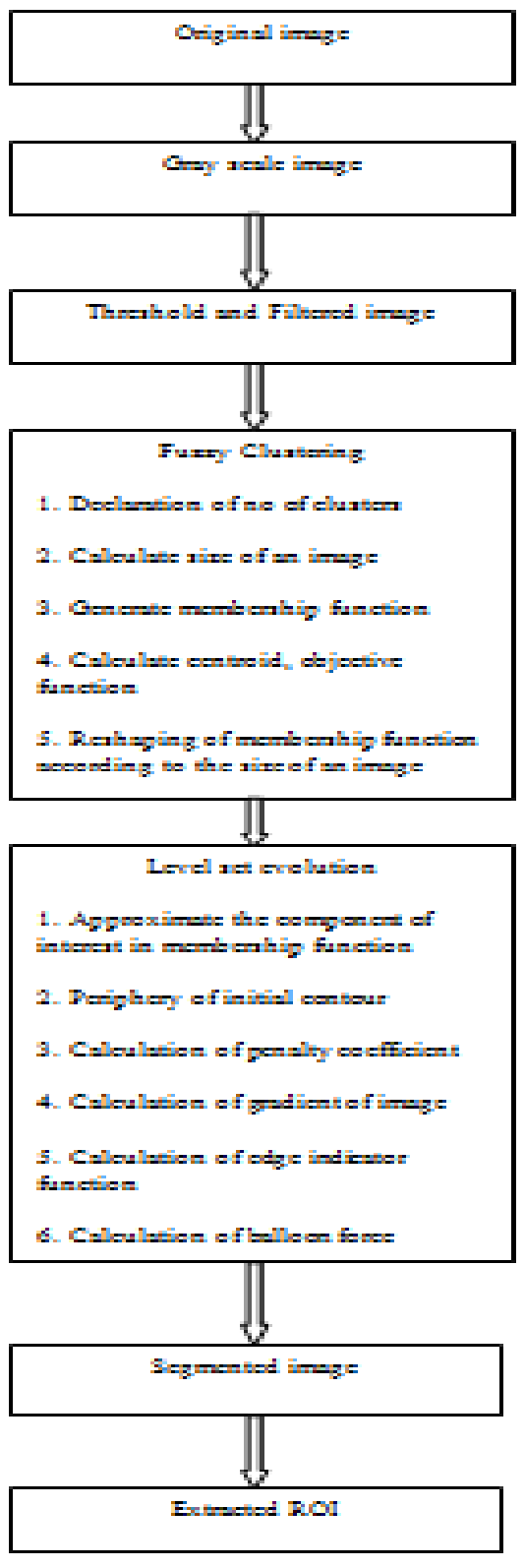

Fig.1: Shows the methodology for Fuzzy with Level Set

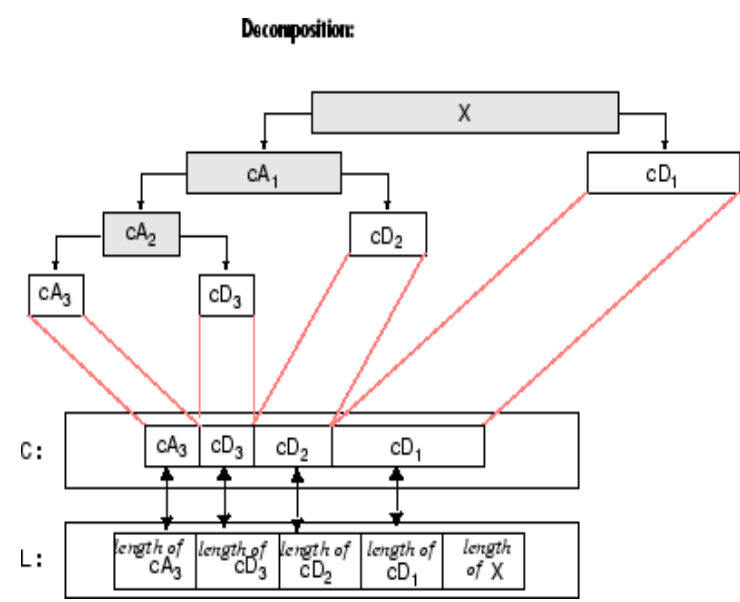

Fig.2: Shows the wavelet decomposition of an image $X$
The 2D-DWT is nowadays established as a key operation in image processing. It is multi-resolution analysis and it decomposes images into wavelet coefficients and scaling function. In Discrete Wavelet Transform, signal energy concentrates to specific wavelet coefficients. This characteristic is useful for compressing images. A 2-D DWT can be seen as a 1-D wavelet scheme which transform along the rows and then a 1-D wavelet transform along the columns. Then the contour is initialized and generates rotationally symmetric Gaussian LPF to further regularize the contour function. In this region based active contour model is used, which utilizes a signed pressure force (SPF) function based on a traditional active contour method. A region-based model is not sensitive to initialization of the level set function and recognizes object's boundaries efficiently [35]. A curve $\mathrm{c}$ in $\Omega$ is represented by a level set function $\phi: \Omega \rightarrow R$, which is zero $\phi=0$ at object boundary in image I. In particular, the evolution of $\varnothing$ is totally determined by the numerical level set function as:

$$
\phi(\mathrm{x}, \mathrm{t}=0)=-\phi \quad \begin{array}{ll}
\mathrm{x} \varepsilon \Omega_{0}-\partial \Omega_{0} \\
0 & \mathrm{x} \varepsilon \partial \Omega_{0}
\end{array}
$$

For a given image I in domain $\Omega$,

$$
\begin{aligned}
& \mathrm{c}_{1}(\phi)=\frac{\int \mathrm{I}(\mathrm{x}) \mathrm{H}(\phi) d x}{\int \mathrm{H}(\phi) d x} \\
& \mathrm{c}_{2}(\phi)=\frac{\int \mathrm{I}(\mathrm{x})(1-\mathrm{H}(\phi)) d x}{\int(1-\mathrm{H}(\phi)) d x}
\end{aligned}
$$

where, $\mathrm{H}(\phi)$ is the Heaviside function.

The SPF function has values in the range $[-1,1]$. It modulates the signs of the pressure forces inside and outside the region of interest so that the contour shrinks when outside the object, or expands when inside the object. The mathematical formulation of the SPF function is as follows:

$$
\operatorname{spf}(I(x))=\frac{I(x)-\frac{c_{1}+c_{2}}{2}}{\max \left(\left|I(x)-\frac{c_{1}+c_{2}}{2}\right|\right)}
$$

Where, $\mathrm{c} 1$ and $\mathrm{c} 2$ are the average intensities inside and outside the contour.

By replacing the edge indicator function shown in equation (8) with the sign pressure force function we get the following formulation:

$$
\frac{\partial \phi}{\partial t}=\operatorname{spf}(\mathrm{I}(\mathrm{x}))|\nabla \phi|\left(\operatorname{div}\left(\frac{\nabla \phi}{|\nabla \phi|}\right)+\mathrm{v}\right)+\nabla \operatorname{spf}(\mathrm{I}(\mathrm{x}) \cdot \nabla(\phi)
$$

where, $v$ is the balloon force, $\operatorname{div}\left(\frac{\nabla \phi}{|\nabla \phi|}\right)$ approximate the mean curvature $\mathrm{k}$.

Hence, the contour is updated according to the magnitude of its gradient, signed pressure force (SPF) and balloon force [26].

In this method, the wavelet transform is used to preserve the edge information of the tumor from the mammogram image also by removing the noise areas of the image. Noise in the 
acquired mammogram image is suppressed by smoothen the image based on the threshold technique of Discrete Wavelet Transform and then reconstructing the original image by taking the inverse of Wavelet Transform. The methodology for mammogram image segmentation using wavelet with level set is shown in Fig: 3

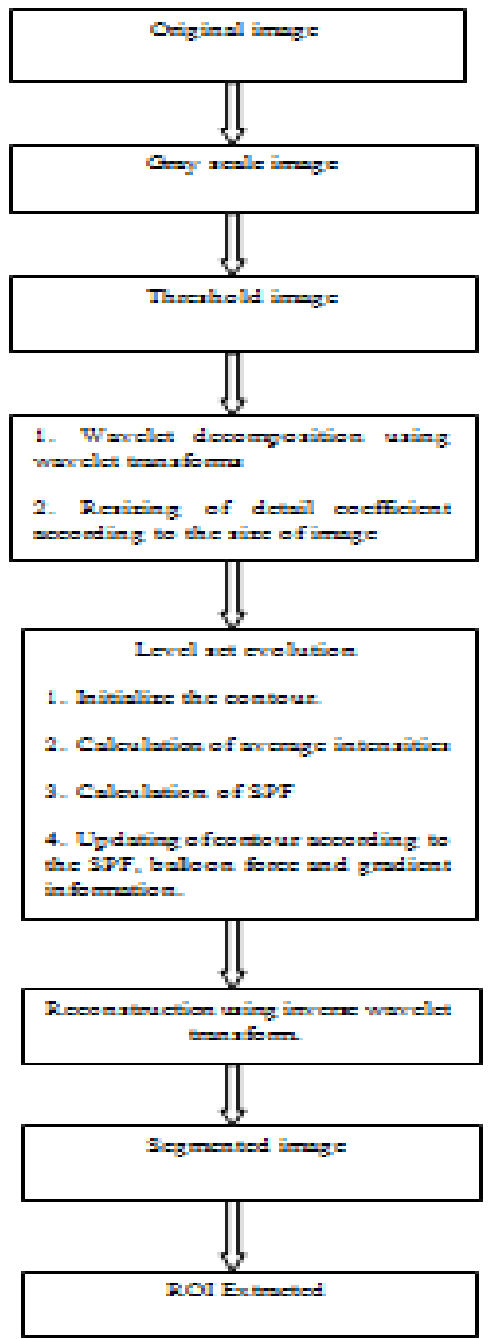

Fig. 3: Shows methodology for Wavelet with level set

\section{EXPERIMENTAL RESULTS}

This section is dividing into three parts. The first part consists of results obtained from mammogram image segmentation using fuzzy level set. In second part the results obtained from mammogram image segmentation using wavelet with level set. Finally in third part, parameters evaluated to compare the performance of these two methods. The proposed approach is applied to a database of some mammographic images, originating from mini-MIAS database and implemented in MATLAB. Results show that proposed approach gives a satisfactory detection performance.

\subsection{Results using Fuzzy with level set}

It is basically used to evaluate the usefulness of an initial fuzzy clustering for level set segmentation and adopted the fast level set algorithm for the curve optimization. Fig. 4 and Fig. 5 show the original image and pre-processed image respectively. Due to weak boundaries and strong background noise, manual initialization did not lead to an optimal level set segmentation. Fuzzy clustering attracts the dynamic curve quickly to the boundaries of interest. Then, these improvements are used to incorporate fuzzy clustering into level set segmentation for an automatic parameter configuration and leads to fast implementation of level set algorithm. The image is segmented using fuzzy level set which is shown in Fig. 6 and the ROI is extracted manually as shown in Fig. 7

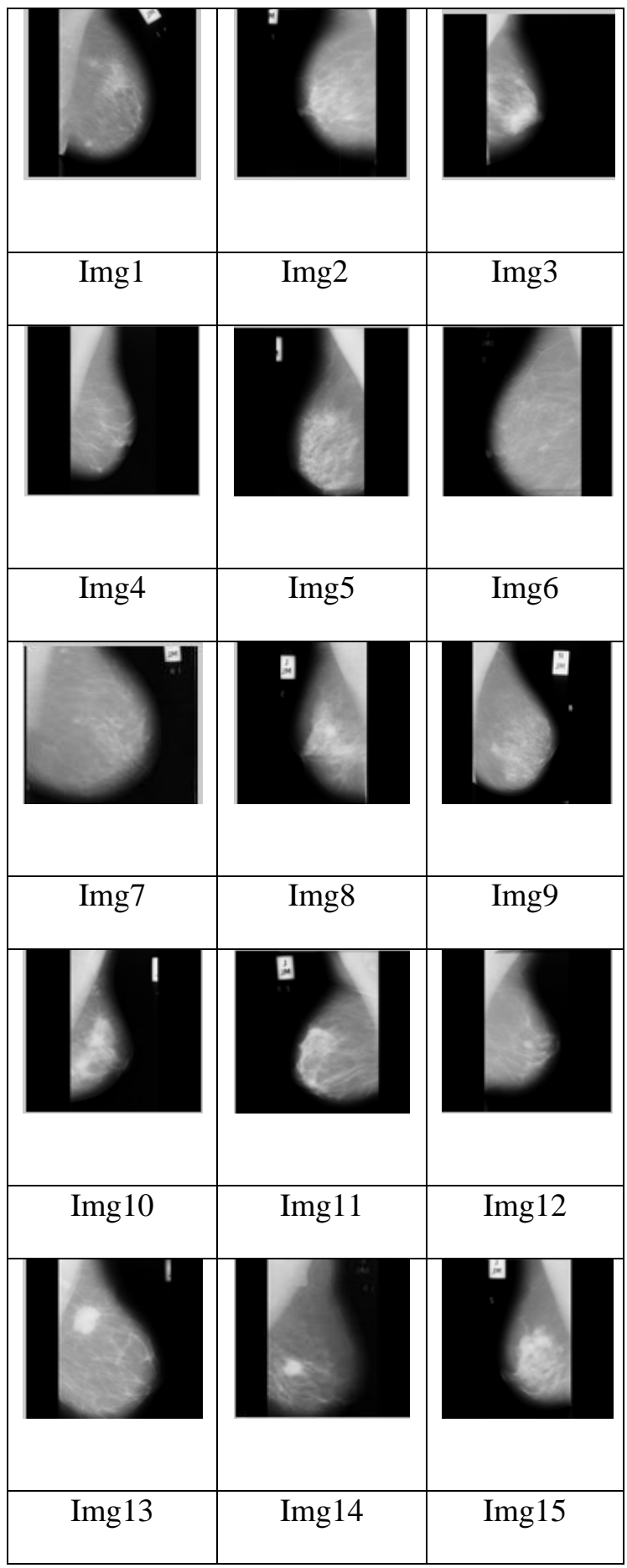

Fig. 4: Original image 


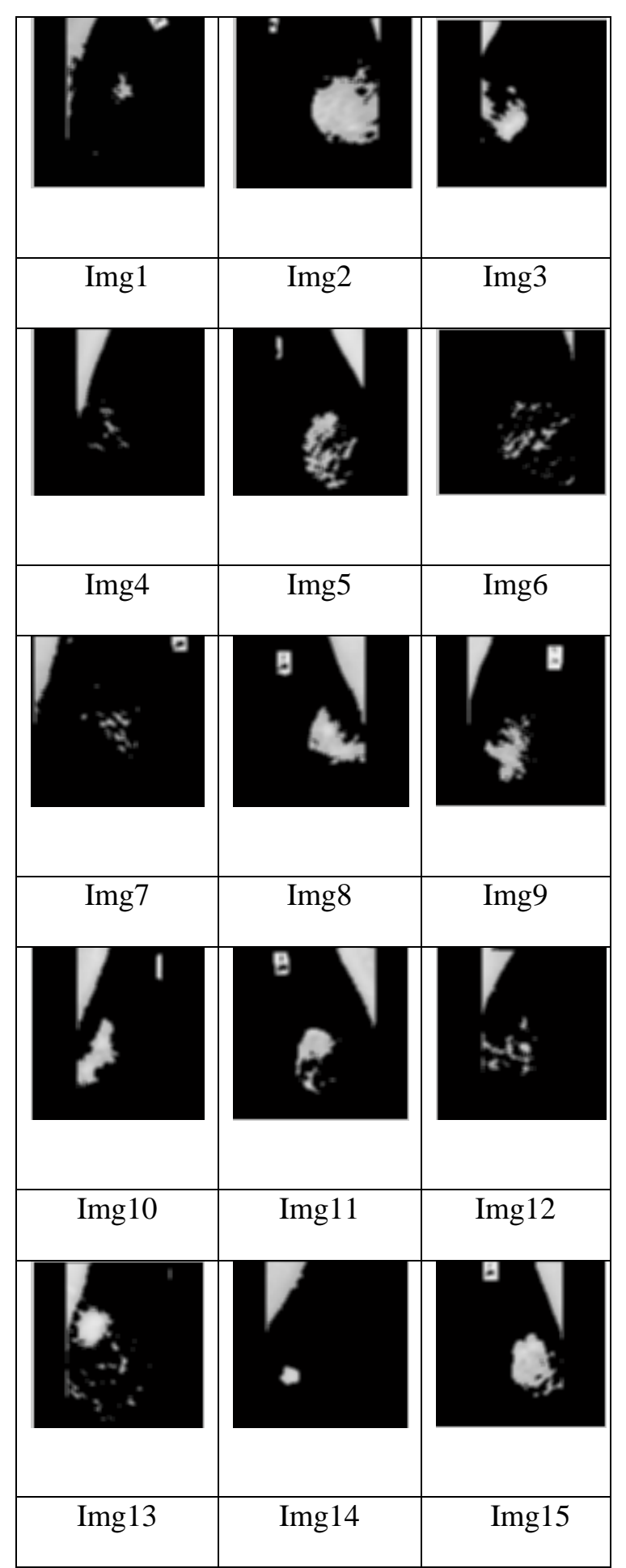

Fig. 5: Pre-processed images

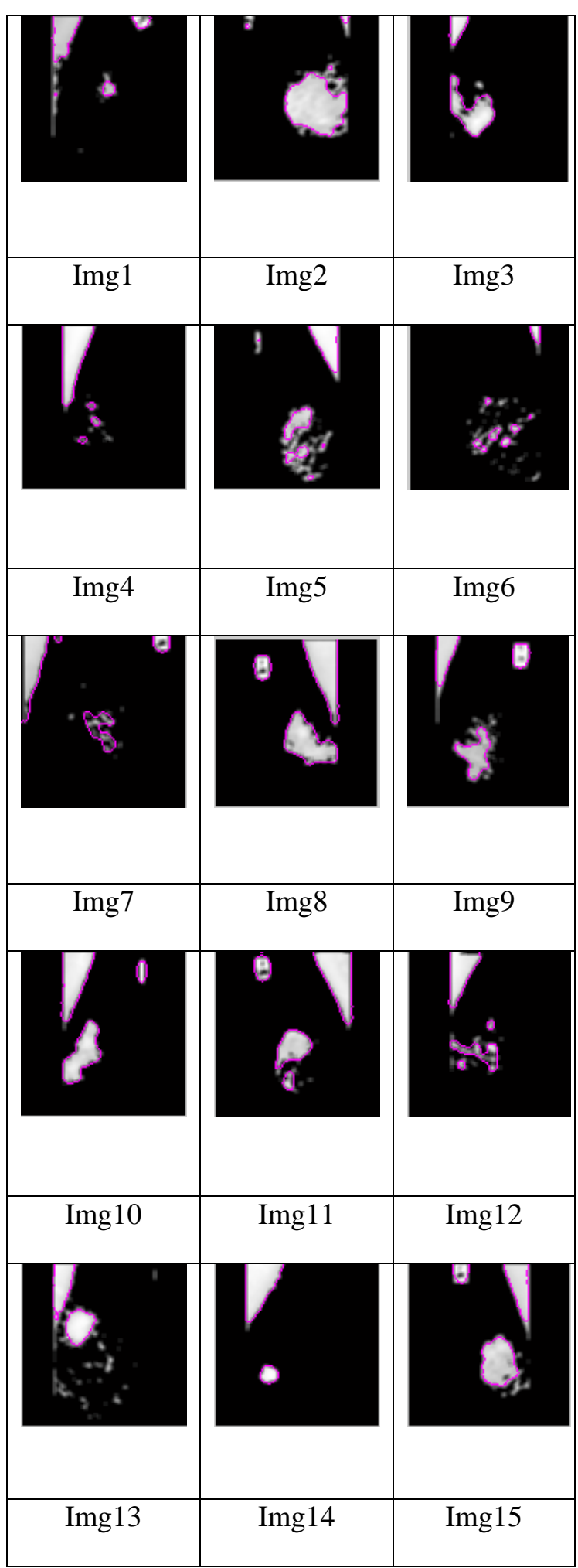

Fig. 6: Segmented images using Fuzzy with level set 


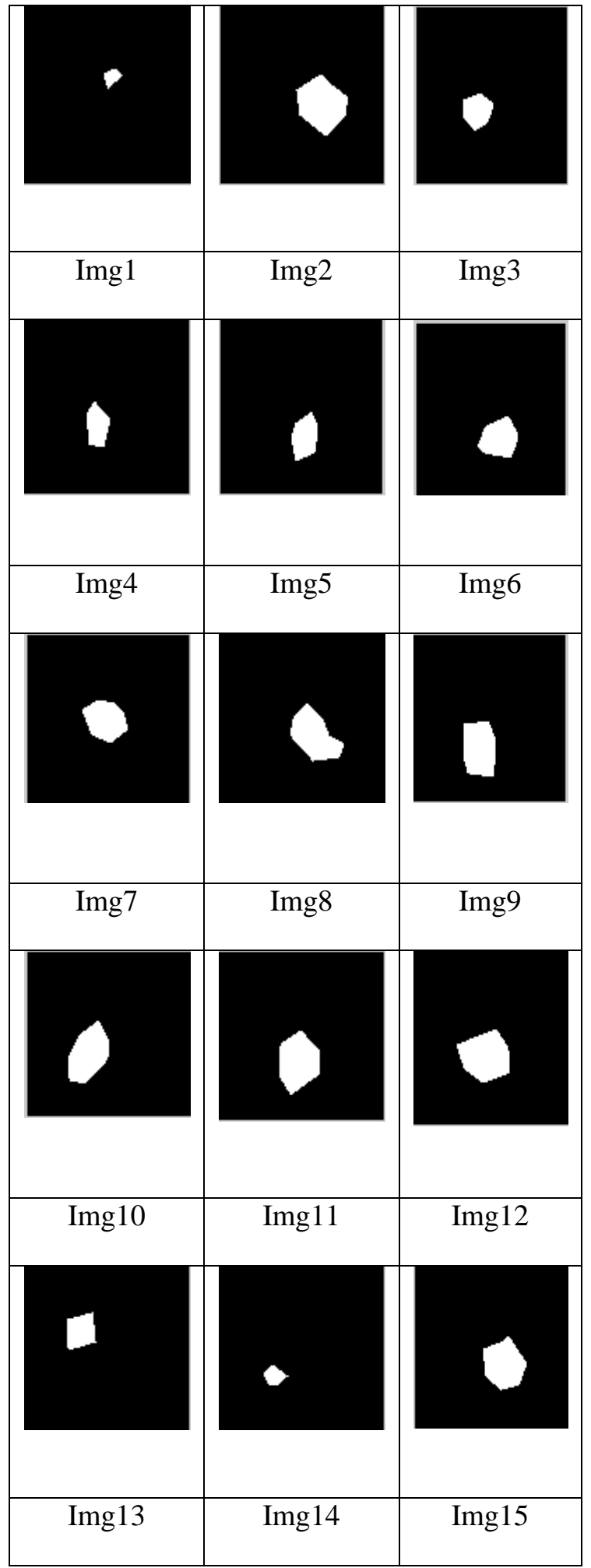

Fig. 7: Extracted ROI

\subsection{Results using wavelet with level set}

The wavelet transform is important to provide a compact description of images that are limited in time and it is very useful in description of edges and line that are highly localized. In wavelet with level set method first, apply wavelet transform on the mammogram image to obtain wavelet decomposed image resulting in four sub bands. These are the LL (lower resolution version of image), LH (horizontal edge data), HL (vertical edge data), \& HH(diagonal edge data) sub bands representing approximation, horizontal, vertical and diagonal components in the form of coefficients, respectively. LL sub band contains low level and the other three (LH, HL, and $\mathrm{HH}$ ) contain high level details. After discrete wavelet transform contour is initialized as shown in Fig.8. Now the contour is updated. Now image is segmented using wavelet with level set as shown in Fig. 9. ROI is extracted manually as shown in Fig. 10.

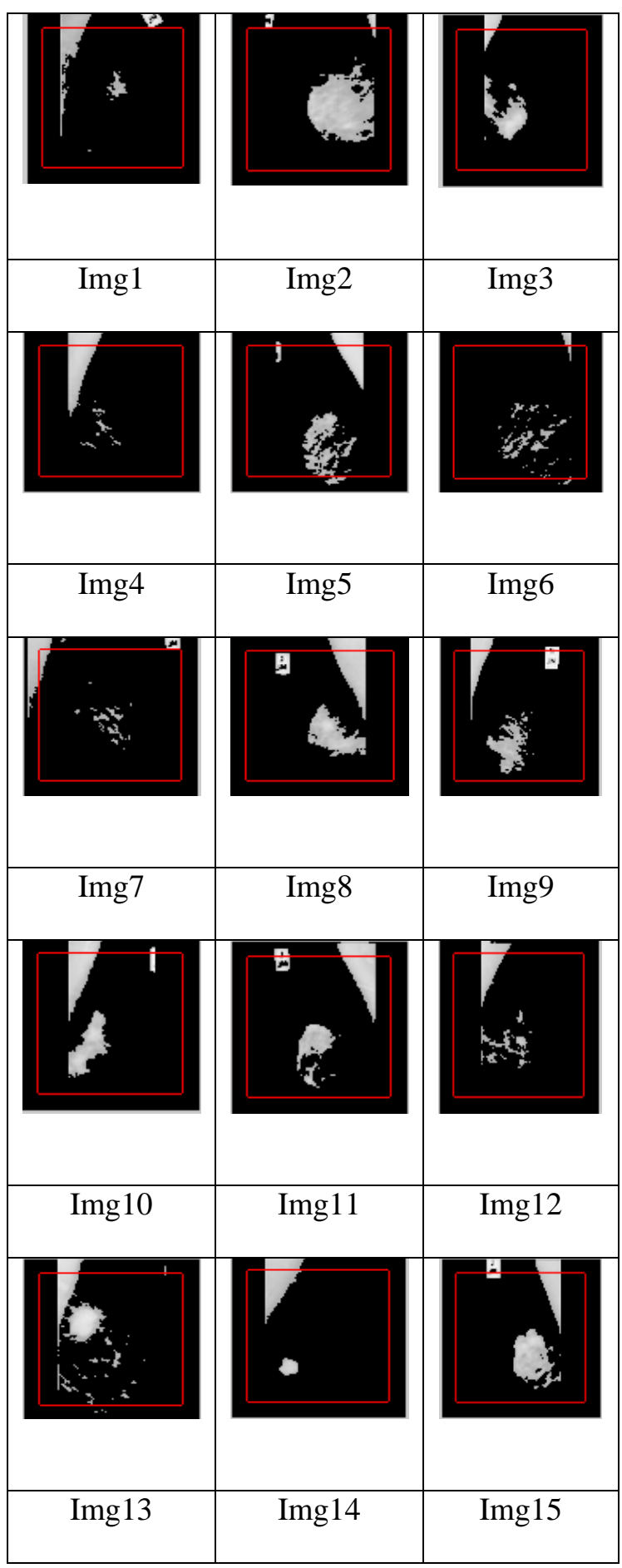

Fig. 8: Threshold image with initial contour 


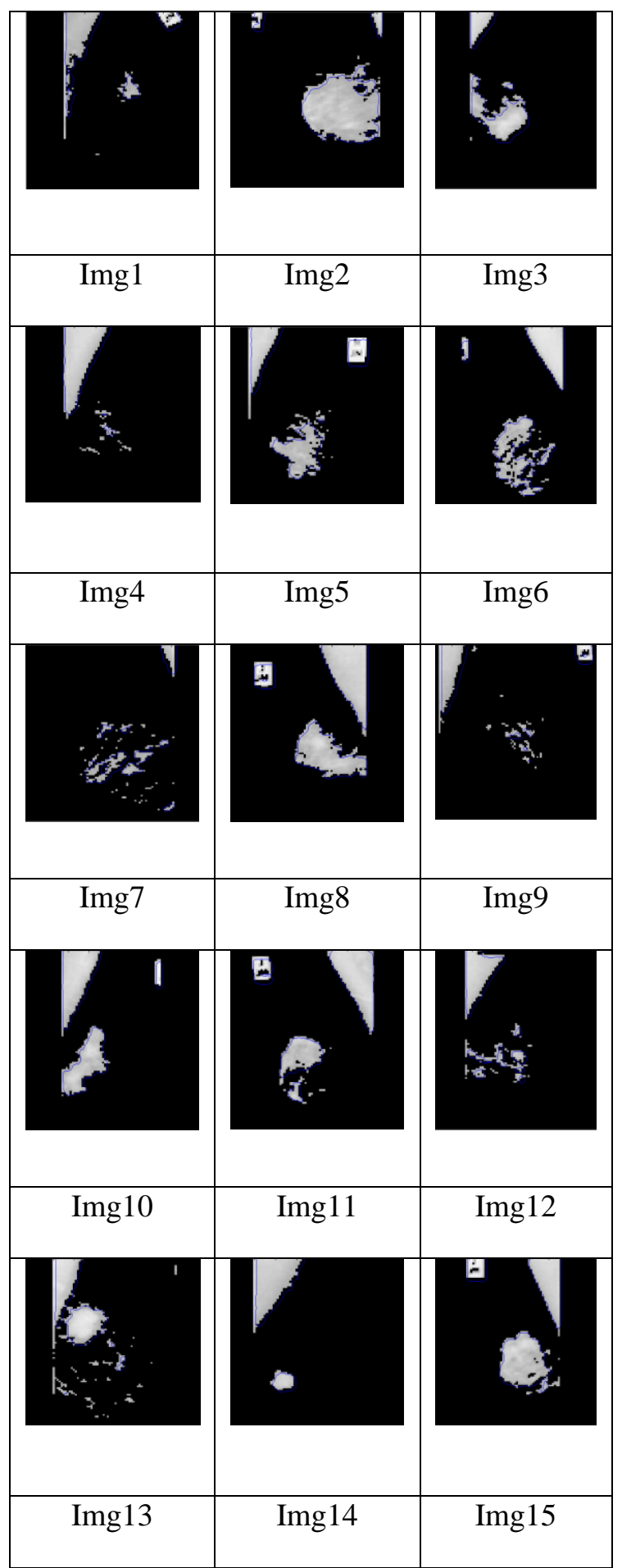

Fig. 9: Segmented image using wavelet with level set

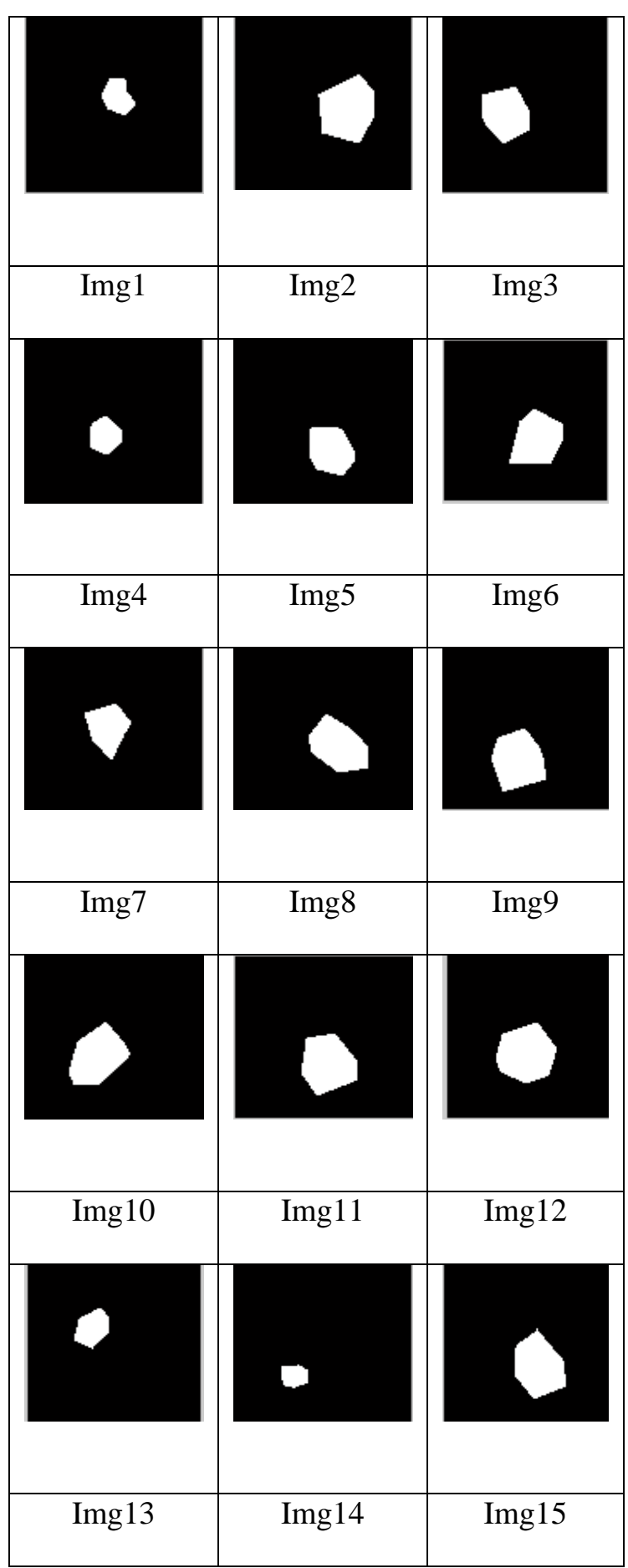

Fig.10: Extracted ROI

\subsection{Parameters Evaluation}

Finally, the parameters are evaluated to compare the performance of segmentation methods.

1. PSNR (peak signal to noise ratio)

PSNR is most commonly used to measure the quality of reconstruction of loss compression.

PSNR can be defined by: 
$P S N R=10 \log _{10}\left(\frac{M A X_{I}^{2}}{M S E}\right)$

2. MSE (mean square error)

For a noise-free $\mathrm{m} \times \mathrm{n}$ monochrome image $\mathrm{I}$ and its noisy approximation $\mathrm{K}, \mathrm{MSE}$ is defined as:

$$
M S E=\frac{1}{m n} \sum_{i=0}^{m-1} \sum_{j=0}^{n-1}[I(I, j)-K(I, j)]^{2}
$$

The MSE and the PSNR are the two error metrics used to compare two image compression qualities. The MSE represents the cumulative squared error between the compressed and the original image, whereas PSNR represents a measure of the peak error. Then the MSE and PSNR is calculated for each image using fuzzy level set and wavelet with level set segmentation methods shown in Table.1.The PSNR values calculated using wavelet with level set is higher as compared to fuzzy with level set and the MSE calculated using wavelet with level set is lower. Thus the lower the value of MSE, the lower the error and high value of PSNR indicates the quality of image.

PSNR is used more often, since it is a logarithmic measure and human brains seem to respond logarithmically to intensity. Edges are the important parameters in consideration of the peak signal-to-noise ratio values. PSNR could be further increased by considering the background details and some high frequency components. Various parameters are calculated to evaluate the performance of proposed methods. The comparisons of different images selected are shown in Table 1.

To proceed towards the area measurement, first separate out ROI from the segmented image. Then extracted area is computed and compared with standard area of mini-MIAS database, whereas the variation between standard database area and segmented area using both methods termed as error value. Error percentage is the ratio of error value to standard area. Error value and error percentage values should be low, as low values of these indicate that system is corresponding well with standard database area and performing well. The simulation results obtained are tabulated in Table.2

TABLE 1: Different parameters for mammogram images using both fuzzy with level set and wavelet with level set

\begin{tabular}{|c|c|c|c|c|}
\hline & Fuzzy with level set & Fuzzy with level set & Wavelet with level set & Wavelet with level set \\
\hline S.No & MSE(mean square error) & $\begin{array}{l}\text { PSNR(peak signal to noise } \\
\text { ratio) }\end{array}$ & MSE(mean square error) & $\begin{array}{l}\text { PSNR(peak signal to } \\
\text { noise ratio) }\end{array}$ \\
\hline 1 & $7.9196 \mathrm{e}+03$ & 9.1437 & $5.9991 \mathrm{e}+03$ & 10.3499 \\
\hline 2 & $8.2542 \mathrm{e}+03$ & 8.9641 & $3.8509 \mathrm{e}+03$ & 12.2751 \\
\hline 3 & $4.8151 \mathrm{e}+03$ & 11.3047 & $2.6611 \mathrm{e}+03$ & 13.8802 \\
\hline 4 & $6.5521 \mathrm{e}+03$ & 9.9390 & $4.1808 \mathrm{e}+03$ & 11.9182 \\
\hline 5 & $7.6461 \mathrm{e}+03$ & 9.9264 & $3.9995 \mathrm{e}+03$ & 12.1107 \\
\hline 6 & $1.0059 \mathrm{e}+04$ & 8.1053 & $9.0068 \mathrm{e}+03$ & 8.5851 \\
\hline 7 & $1.2886 \mathrm{e}+04$ & 7.0295 & $1.0930 \mathrm{e}+04$ & 7.7445 \\
\hline 8 & $7.9698 \mathrm{e}+03$ & 9.1163 & $3.3495 \mathrm{e}+03$ & 12.8810 \\
\hline 9 & $8.7961 \mathrm{e}+03$ & 8.6879 & $5.3970 \mathrm{e}+03$ & 10.8093 \\
\hline 10 & $5.9251 \mathrm{e}+03$ & 10.3841 & $2.1193 e+03$ & 14.8690 \\
\hline 11 & $8.0675 \mathrm{e}+03$ & 9.0634 & $4.1851 \mathrm{e}+03$ & 11.9137 \\
\hline 12 & $6.5841 \mathrm{e}+03$ & 9.9458 & $4.5875 \mathrm{e}+03$ & 11.5150 \\
\hline 13 & $1.0894 \mathrm{e}+04$ & 7.7591 & $7.4151 \mathrm{e}+03$ & 9.4296 \\
\hline 14 & $6.6764 \mathrm{e}+03$ & 9.8554 & $4.4159 \mathrm{e}+03$ & 11.6806 \\
\hline 15 & $7.5549 \mathrm{e}+03$ & 9.3485 & $3.1966 \mathrm{e}+03$ & 13.0839 \\
\hline
\end{tabular}


TABLE 2: Performance analysis of image segmentation techniques

\begin{tabular}{|c|c|c|c|c|c|c|c|}
\hline & & \multicolumn{3}{|c|}{ Fuzzy with level set } & \multicolumn{3}{|c|}{ Wavelet with level set } \\
\hline S. No. & $\begin{array}{c}\text { Area using } \\
\text { standard } \\
\text { datbase } \\
(m m)^{2}\end{array}$ & $\begin{array}{c}\text { Segmented } \\
\text { area }(m m)^{2}\end{array}$ & Error Value & Error \% & $\begin{array}{l}\text { Segmented area } \\
(\mathrm{mm})^{2}\end{array}$ & Error Value & Error \% \\
\hline 1 & 34.3910 & 25.3618 & 9.02 & 0.26 & 31.5498 & 2.84 & 0.08 \\
\hline 2 & 114.350 & 91.1986 & 23.15 & 0.20 & 104.985 & 9.36 & 0.08 \\
\hline 3 & 93.2961 & 61.1626 & 31.13 & 0.33 & 82.9281 & 10.36 & 0.11 \\
\hline 4 & 47.6000 & 35.1050 & 12.49 & 0.26 & 37.7230 & 9.87 & 0.20 \\
\hline 5 & 62.9510 & 39.2402 & 23.71 & 0.37 & 50.8873 & 12.06 & 0.19 \\
\hline 6 & 137.564 & 65.0335 & 72.53 & 0.52 & 98.9633 & 38.60 & 0.28 \\
\hline 7 & 86.7510 & 59.4851 & 27.26 & 0.31 & 64.3343 & 22.41 & 0.25 \\
\hline 8 & 129.591 & 75.8030 & 53.78 & 0.41 & 93.8017 & 35.78 & 0.27 \\
\hline 9 & 86.7510 & 74.8800 & 11.87 & 0.13 & 81.5150 & 5.23 & 0.06 \\
\hline 10 & 171.836 & 77.6623 & 94.17 & 0.54 & 100.198 & 71.63 & 0.41 \\
\hline 11 & 100.079 & 79.3135 & 20.76 & 0.20 & 92.8497 & 7.22 & 0.07 \\
\hline 12 & 129.591 & 86.6468 & 42.94 & 0.33 & 117.200 & 12.39 & 0.09 \\
\hline 13 & 57.5960 & 37.5296 & 20.06 & 0.34 & 42.6466 & 14.94 & 0.25 \\
\hline 14 & 47.6000 & 34.8075 & 12.79 & 0.26 & 43.0681 & 4.53 & 0.09 \\
\hline 15 & 121.856 & 75.8625 & 45.99 & 0.37 & 108.2453 & 13.61 & 0.11 \\
\hline
\end{tabular}

\section{CONCLUSIONS}

Breast cancer is one of the major causes of death among women, so, early diagnosis through regular screening and timely treatment has been known to prevent cancer. In this work, a novel approach to segment the breast cancer masses and calcifications in mammogram images has been presented. Image segmentation is the challenging and active area of research in the field of medical image analysis. The proposed work utilizes fuzzy level set and wavelet with level set for image segmentation. Using this, suspicious breast cancer masses and calcifications in digital mammogram images have successfully segmented and ROI has been extracted manually.

One of the biggest challenges in level set segmentation is the intensive computation. In fuzzy level set and wavelet with level set, parameters are estimated directly from fuzzy clustering and wavelet transform respectively and thus the manual intervention has been reduced and level set evolution has been regularize in order to alleviate the problem of noise sensitivity and weak boundaries.
These improvements leads to fast level set algorithm for mammogram image segmentation.

In wavelet with level set, region based active contour model utilizes an signed pressure force (SPF) function based on a traditional active contour method's region-based model is not sensitive to initialization of the level set function and recognize object's boundaries efficiently and, wavelet transform made the algorithm noise free because wavelet provides frequency information as well as time-space localization. In addition, their multi-resolution character enables us to visualize image at various scales and orientation. Furthermore, after evaluating various parameters wavelet with level set is considered to be better than fuzzy level set, as segmentation of mass area is more effective having less error value and it shows higher PSNR as compared to other methods.

One of the limitation of these methods is accuracy, as the ROI has been extracted manually which results in reduction in accuracy which may be overcome in future by using automatic image segmentation techniques. 


\section{REFERENCES}

[1] R. Ramani, S. Suthanthiravanitha and S. Valarmathy, "A survey of current image segmentation techniques for detection of breast cancer" International Journal of Engineering Research and Applications (IJERA), vol. 2, Issue 5, pp. 1124-1129, 2012.

[2] D.L. Pharm, C. Xu, J. L. Prince, "current methods in medical image segmentation" Annual review of biomedical engineering, 2000.

[3] R. Ramani, Dr. S. Suthanthiravanitha and S.Valarmathy, "A survey of current image segmentation techniques for detection of breast cancer" International Journal of Engineering Research and Applications (IJERA), vol. 2, Issue 5, pp.1124-1129, 2012.

[4] R. Sura. Shareef "Breast cancer detection based on watershed transformation" IJCSI International Journal of Computer Science Issues, vol. 11, Issue 1, no. 1, pp. 237 245, 2014.

[5] H. Moradmand, S. Setayeshi and H. K. Targhi "Comparing methods for segmentation of microcalcification clusters in digitized mammograms" IJCSI International Journal of Computer Science Issues, vol. 8, Issue 6, no.1, pp. 104-108,2011.

[6] Varsha J. Gaikwad "Marker controlled watershed transform in digital mammogram segmentation" International Journal for Research in Applied Science \& Engineering Technology (IJRASET). vol. 3, pp. 18-21, 2015.

[7] S.ong yang Yu and Ling Guan "A CAD system for the automatic detection of clustered micro calcifications in digitized mammogram films" IEEE Transactions on Medical Imaging, vol. 19, no. 2, pp. 115-126,2000.

[8] Cheng, H. D., Shi, X. J., Min, R., Hu, L. M., Cai, X. P., and $\mathrm{Du}, \mathrm{H}$. N."Approaches for automated detection and classification of masses in mammograms"Pattern Recognition 39, 646-668, 2006.

[9] Oliver, A., Freixenet, J., Marti, J., Perez, E., Pont, J., Denton, E., and Zwiggelaar, R. "A review of automatic mass detection and segmentation in mammographic images" Medical Image Analysis.14, 87-110, 2010.

[10] L.P. Clarke, R.P. Velthuizen, M.A. Camacho, J.J. Heine, M. Vaidyanathan, L.O. Hall, R.W. Thatcher and M.L. Silbiger, "MRI Segmentation: Methods and Applications" Magnetic Resonance Imaging, vol. 13, no. 3, pp. 343-368, 1995

[11] Z. A. Jaffery, Zaheeruddin and L. Singh "Performance analysis of image segmentation methods for the detection of masses in mammograms" International Journal of Computer Applications, Vol. 82, no.2, pp. 44-50, 2013.

[12] Dunn, J. 1974 "a fuzzy relative of the iso-dataprocess and its use in detecting compact well separated clusters" J.cybernectics.3, 32-57.

[13] Dominquez, J.Q., Magana, B.O., Januchs, M.G., Ruelas, R., Corona, A.V., Andina, D. "Image segmentation by fuzzy and possibility clustering algorithms for the identification of micro-calcification" Scientia Irenic D. 18,580-589, 2011.

[14] L, Y. C., Tsai, Y.P., Hung, Y.P., and Shih, Z.C., "Comparison between immersion based and toboggan based watershed image segmentation"IEEE Trans. Image Processing.15, 632-640, 2006.

[15] Vincent, L., Soille, P. "Watersheds in digital spaces: an efficient algorithm based on immersion simulations"IEEE Trans. Pattern and Machine Intelligence.13, 583-598, 1991.

[16] Adams, R., and Bishop, L, "Seeded region growing".IEEE Trans. Pattern and Machine Intelligence.16 (6), 641-647, 1994.

[17] S. Sasikala, M. Ezhilarasi, P.Sudharsan, C.L.Yashwanthi Sivakumari " Performance analysis of various segmentation techniques in breast mammogram images" International conference on intelligent computing Applications, 2014.

[18] Mohamed Ali HAMDI, K. S. Ettabaa and Mohamed Lamine HARABI “A new Mammography segmentation technique based on watershed, wavelet and curve let transform." Computers, Automatic Control Signal Processing and Systems Science.

[19] S. Dalmiya, A. Dasgupta and S. K. Datta "Application of wavelet based K-means algorithm in mammogram segmentation" International Journal of Computer Applications,vol. 52, no.15, 2012.

[20] Gokila Deepa.G "Mammogram image segmentation using Fuzzy Hybrid with Particle Swarm Optimization(PSO)" International Journal of Engineering and Innovative Technology, vol. 2, 2011

[21] M. Cass, A. Wit kin, and D. Terzopoulos, "Snakes: active contour models," International Journal of Computer Vision, vol. 1, no. 4, pp. 321-331, 1988.

[22] V. Casella's, R. Kimmel, and G. Spiro, "Geodesic active contours," International Journal of Computer Vision, vol. 22, no. 1, pp. 61-79, 1997.

[23] C. Li, C. Xu, C. Gui, and M. D. Fox, "Level set evolution without re-initialization: a new variation formulation," in Proceedings of the IEEE Computer Society Conference on Computer Vision and Pattern Recognition , pp. 430-436, 2005.

[24] N. Paragios and R. Deriche, "Geodesic active regions and level set methods for supervised texture segmentation" International Journal of Computer Vision, vol.46, no.3, pp.223-247, 2002

[25] T. F. Chan and L. A. Vase, "Active contours without edges," IEEE Transactions on Image Processing, vol. 10, no. 2, pp. 266-277, 2001.

[26] K. Zhang, L. Zhang, H. Song, and W. Zhou, "Active contours with selective local or global segmentation: a new formulation and level set method," Image and Vision Computing, vol. 28, no. 4, pp. 668-676, 2010.

[27] D. Mumford and J. Shah, "Optimal approximations by piecewise smooth functions and associated variation problems," Communications on Pure and Applied Mathematics, vol. 42, no. 5, pp. 577-685, 1989.

[28] J. Lie, M. Lysaker, and X.-C. Tai, "A binary level set model and some applications to Mumford-Shah image segmentation," IEEE Transactions on Image Processing, vol. 15, no. 5, pp. 1171-1181, 2006. 
[29] L. A. Vase and T. F. Chan, "A multiphase level set framework for image segmentation using the Mumford and Shah model," International Journal of Computer Vision, vol. 50, no. 3, pp. 271-293, 2002.

[30] D. Cremer's, "A multiphase level set framework for motion segmentation," in Scale Space Methods in Computer Vision: 4th International Conference, Scale Space 2003 Isle of Skye, UK, June 10-12, 2003Proceedings, vol. 2695 of Lecture Notes in Computer Science, pp. 599-614, Springer, Berlin, Germany, 2003.

[31] R. Ranford, "Region-based strategies for active contour models," International Journal of Computer Vision, vol. 13,no. 2, pp. 229-251, 1994.

[32] C. Li, C.-Y. Kao, J. C. Gore, and Z. Ding, "Implicit active contours driven by local binary fitting energy," in Proceedings of the IEEE Computer Society Conference on Computer Vision and Pattern Recognition (CVPR '07), pp. 1-7, Washington, DC, USA, June 2007.

[33] H. Jiang, R. Feng, and X. Gao, "Level set based on signed pressure force function and its application in liver image segmentation," Wuhan University Journal of Natural Sciences,vol. 16, no. 3, pp. 265-270,2011.

[34] J. Gomes and O. Faugeras, "Reconciling distance functions and level sets" Journal of Visual Communication and Image Representation, vol. 11, no. 2, pp. 209-223, 2000.

[35] F. Akram, J. H. Kim, C-G Lee and K. N. Choi "Segmentation of Regions of Interest Using Active Contours with SPF Function", 2015.

[36] B. N. Li, C.K. Chui and S. H. Ong, "Integrating FCM and level set for liver tumor segmentation, in: proceedings of the $13^{\text {th }}$ international conference on biomedical engineering, IFMBE Proceedings, vol. 23, pp. 202-205, 2009.

[37] J. S. Suri, K. Liu, S. Singh, S. N. laxminarayan, X. Zeng and L. Reden, "Shape recovery algorithm using level set in 2-D/3-D medical imaginary: a state of art review" IEEE Transactions on medical imaging 22, 773$776,2003$.
[38] N. Paragios, "A level set approach for shape -driven segmentation and tracking of left ventricle"IEEE Transaction on medical imaging 22,773-776, 2003.

[39] I. M. Mitchell, "The flexible, extensible and efficient toolbox of level set methods" Journal of scientific computing 35, 300-329, 2008.

[40] J. S. Suri, “ Two dimensional fast magnetic resonance brain segmentation" IEEE Engineering in Medicine and Biology Magazine 20, 84-95, 2001

[41] S. Ho, E. Bullitt and G. Gerig, "level set evolution with region competition: automatic 3-D segmentation of brain tumours" in: Proceedings of the international conference on pattern Recognition, 532-535,2002.

[42] P. A. Yushkevich, J. Piven, H. C. Hazlett, R. G. Smith and S. Ho, J. C. Gee, et al, "user guided 3D active contour segmentation of anatomical structures: significantly improved efficiency and reliability", NeuroImage, vol. 31, pp. 1116-1128, 2006.

[43] Bing, N. Li, C. K. Chui, S. Chang and S. H. Ong "Integrating spatial fuzzy clustering with level set methods for automated medical image segmentation" Computers in Biology and Medicine, 2011.

[44] R. Ramani, S. Suthanthiravanitha and S. Valarmathy “ A survey of current image segmentation techniques for detection of breast cancer" International Journal of Engineering Research and Applications (IJERA), vol. 2, Issue 5, pp.1124-1129, 2012.

[45] D. L. Pharm, C. Xu and J. L. Prince "current methods in medical image segmentation" Annual review of biomedical engineering, 2000.

[46] K. Levinski, A. Sourin and V. Zagorodnav "Interactive surface-guided segmentation of brain MRI data" Computers in Biology and Medicine, pp. 1153-1160, 2009.

[47] W. Cai, S. Chen and D. Zhang "Fast and robust fuzzy c-means clustering algorithms incorporating local information for image segmentation" Pattern recognition, pp. $825-838,2007$ 